\title{
Clinical governance and clinical competence to support new scenarios and role of internal medicine
}

\author{
Antonino Mazzone, ${ }^{1}$ Mauro Campanini, ${ }^{2}$ Stefano De Carli, ${ }^{3}$ Micaela La Regina, ${ }^{4}$ Andrea Montagnani,,${ }^{5}$ \\ Laura Morbidoni, ${ }^{6}$ Roberta Re, ${ }^{2}$ Alba Sciascera, ${ }^{1}$ Ilario Stefani, ${ }^{1}$ Giancarlo Tintori, ${ }^{7}$ Roberto Nardi ${ }^{8}$ for the \\ FADOI Groups of Clinical Governance
}

${ }^{1}$ Medical Area Department, Hospital of Legnano (MI); ${ }^{2}$ Medical Department, University Hospital Maggiore della Carità, Novara; ${ }^{3}$ Division of Internal Medicine 2, University Hospital of Udine; ${ }^{4}$ Division of Internal Medicine, Sant'Andrea Hospital, La Spezia; ${ }^{5}$ Division of Internal Medicine, Misericordia Hospital, Grosseto; ${ }^{6}$ Division of Internal Medicine, Senigallia Hospital, Ancona; ${ }^{7}$ Division of Emergency Medicine, University Hospital of Pisa; ${ }^{8}$ Division of Internal Medicine, Maggiore Hospital, Bologna, Italy

\begin{abstract}
The complex patient, who has often multiple, chronic and progressive disorders, who has undergone polytherapy, should be evaluated totally with respect not only to medical side, but also to psychological and clinical side. The shortage of specialists in disciplines that require technical skills, obtained by training and performing a sufficient number of annual procedures, contributes to the need for reorganizing health care; in this background the Internist devolves less time to charitable activities in favor of competences related to the processes. The knowledge of the clinical governance (CG) should be the common heritage of all the actors of the health system, that need to be made up of professionals able to coordinate and make easy the implementation and the spread the CG culture. At least initially we propose to focus the testing strictly on the medical department. As already mentioned above, the natural Internist predisposition, cultural and training, leads him to a multidisciplinary vision of medicine that allows acquiring more easily the tools that make up the structure of CG, being able to facilitate the application. The acquisitions of professional competence and clinical governance play a key role in the Internist culture. The purpose of an Internist with professional skills and managerial capacity, is to act within the department to facilitate and simplify the horizontal interaction among other similar corporate structures and to help the Management to improve structural and clinical appropriateness in Hospital and to better the relations between hospital and territory, identifying the critical issues and the possible solutions.
\end{abstract}

\section{Introduction}

The concept of complexity in medicine is often the subject matter of speaking ${ }^{1-3}$ although for decades

Correspondence: Giancarlo Tintori, Ospedale Nuovo Santa Chiara, Azienda Ospedaliero Universitaria Pisana, via Paradisa 2, 56100 Pisa, Italy.

Tel.: +39.050.997827 - Fax: +39.050 .997374$

E-mail: giancarlotintori@hotmail.it

Key words: Internal medicine; clinical governance; clinical competence; complex patient.

Conflict of interest: the authors declare no conflict of interest.

See online Appendix for Members of the FADOI Groups of Clinical Governance.

Received for publication: 9 May 2015.

Revision received: 14 August 2015.

Accepted for publication: 18 August 2015.

This work is licensed under a Creative Commons Attribution NonCommercial 4.0 License (CC BY-NC 4.0).

CCopyright A. Mazzone et al., 2016

Licensee PAGEPress, Italy

Italian Journal of Medicine 2016; 10:92-95

doi:10.4081/itjm.2015.514 its meaning has been misinterpreted as if this problem could be resolved by a growing number of medical and surgical subspecialties. The complex patient, suffering from multiple disorders, often chronic and progressive and treated with polytherapy, should be globally evaluated taking into account medical, psychological and social problems. ${ }^{4}$ Nevertheless the traditional concept of an holistic approach, considered synonymous of Internal Medicine, now appears insufficient and restrictive. ${ }^{5}$ In the era of spending review you need to be efficient and effective by rediscovering the clinical method as an indispensable instrument and by applying scientific evolution in the patient's personalized medical sciences. Historically, medicine has evolved gradually transforming the diagnostic and therapeutic approach based initially on assumptions, mainly the result of experience and expert opinion, or arising from assessments of qualitative criteria to an increasingly quantitative or measurable approach [evidence-based medicine (EBM)]. ${ }^{6}$ However in the real world the diagnostic and therapeutic algorithms deriving from evidencebased medicine could be applied only in $20 \%$ of patients. A possible solution could be the application of the so-called medicine of the 4 P, i.e., Personalized (based on individual characteristics), Predictive (based on analysis of the patient to get an accurate 
assessment of the risk of contracting diseases), Preventive (is based on the above, allows to field preventive measures in the form of screening tests and/or specific interventions), Participatory (individual participation of the patient is essential in order to achieve the above points). ${ }^{7}$ Compared to the past, however, the physician should take into account not only the interest of patient but even the economic aspects deriving from that care, considering that an increase in health care spending is not necessarily correlated to a better healthcare system and in fact beyond a certain commitment of resources, you reach a plateau beyond which further investments do not match the significant improvements in outcomes such as survival and quality of care. This raises the need for a new method of interpreting the medicine that combines scientific progress, represented by evidence-based medicine, research innovation (such as algorithms or off-label treatments for rare diseases where there is no EBM), clinical and economic knowledge. The internist for training and attitudes is the specialist more than other that best interprets this approach to health care management.

\section{New perspectives of Internal Medicine}

In Italy the number of doctors is progressively diminishing due to the retirement of the age groups that have started their profession in the ' $70 \mathrm{~s}$ and ' $80 \mathrm{~s}$ and that do not have had an adequate replacement. Of particular relevance is a significant reduction in the training of specialists in medical areas such as endocrinology, gastroenterology, etc., that in the near future will be inadequate to the needs of the healthcare system. The shortage of specialists in disciplines that require technical skills, obtained by training and running a sufficient number of annual performance, contributes to the need for reorganizing health care; in this background the Internist devolves less time to charitable activities in favor of competences related to the processes. Therefore, it is expected a gradual reduction in specialized beds in favor of those in the medical area, a situation already partly observed, that determines an engagement of the internist in disciplines (see diabetes) that until a few years ago were reserved to specialists.

A typical example of a patient who may benefit from this new organization is the patient affected by chronic obstructive pulmonary disease with recurrent complications, complex drug therapies and whose cause of death is rarely respiratory; this patient should be managed by a multidisciplinary team in a medical properly organized ward whereas the pulmonologist should play a fundamental role when there is need of specific techniques such as bronchoscopy, thoracoscopy, etc., that can be done as outpatient.

\section{Exceeding the hospital's model for intensity of care}

The impact of chronic diseases on health therefore involves a multidisciplinary approach in which the internist plays a strategic role. The attempt to solve the centrality of the patient using the hospital's model for intensity of care has not achieved the desired results for a variety of reasons, ${ }^{4}$ first of all it is a rigid model which absorbs resources and time just to define the best structure where to admit the patient, moreover it is a system of linear production where it is possible that a patient must return to a previous stage of the care process. It should not be underestimated that the obsolescence of the actual buildings makes difficult the implementation of the model for intensity of care. This model has failed to facilitate the integration between hospital and territory for whose achievement the nurse should be the pivotal figure. The nurse casemanager is a central figure in case of lower level of intensity of care also for improving the interactions between specialists. It should also be pointed out that especially in large hospitals the strategic directions have a tendency to marginalize internal medicine when you have to translate the different guidelines in clinical pathways care related to complex patients, though these subjects are predominantly treated in medical wards, where the effects of organizational choices are more sensitive. This situation is responsible for the underestimation of the pivotal role of the Internal Medicine that is involved only passively in the implementation phase. That causes for example the paradoxical case of patients suffering from heart failure managed in Internal Medicine wards during exacerbations and in Cardiology Department during stable phases for diagnostic and therapeutic purposes and for follow-up.

\section{The clinical governance facilitator}

The knowledge of the clinical governance (CG) should be the common heritage of all actors of the health system, but they need to be made up of professionals able to coordinate, facilitate the implementation and spread the culture of $\mathrm{CG}^{8}$ The $\mathrm{CG}$ facilitator should have a strong connection with the Strategic Department, which is in charge while remaining in staffing plan of the department he belongs to. Currently the figure of the Facilitator has already been included in the Clinical Risk area; in fact some experiences in Italy, especially in Tuscany, have involved doctors and nurses, divided by areas of expertise, dealing with clinical risk and organization of clinical audit to support the Health Department. These experiences have been often unsatisfactory due to their implementation in no 
core departments and to their goals limited to the achievement of budget targets. We propose additional tasks to assign to the Facilitator for promoting the integration of intra-and inter-departmental changes, for implementing the risk management tools, for translating organizational culture and innovations, economic and staff management skills, starting initially within the medical department. As already mentioned above the natural Internist predisposition, culture and training, lead him to a multidisciplinary vision of medicine allowing easier acquisition and application of the clinical governance tools and skills. In addition, the internal medicine department is always represented, forming the core of the hospital system and is the most easily interfaced to the territory.

\section{Role of the clinical governance facilitator in the medical department}

As mentioned before the internist has always been the emblem of the interdisciplinarity, facing daily clinical problems across the board, caring for patients affected by multiple pathologies; this involves the ability to manage interdisciplinarity and summarize the clinical problems through the diffusion and implementation of technical tools (such as care pathways) and organizational issues related to the spread of the departments. This new vision of the internist role should be included in a revolution that witnessed the transition from output (related to wealth) to outcome assessment (needed for resources rationalization). These skills and competencies should be known by all internists, but the facilitator should be a clinician who has developed specific knowledge of CG and works in the medical department in order to solve organizational problems facilitating the spread of CG culture. He should be member of the Department Board that may also change over time, this ideally would favor the spread of CG, but to ensure continuity this role should be assigned to professional with proven expertise in the field. Being a member of the board of the department would have a double benefit: ensure the sharing and the involvement of other technical and health figures that are not necessarily only the head of department and the directors of Complex Units, and operate with a strong mandate, with transparency and effectiveness, helping to give greater dignity and importance to this collegial body. The Facilitator would have the task of co- integrating with the Health Directorate (HD), without replacing the offices in charge, such as that of clinical risk, with high organizational content and with legal implications, in which the purely clinical contribution would be less relevant; this in order to clarify his not unlimited possibilities for action.

First of all, you have established the facilitator tasks in relation to the various branches of the Chambers' tree. For the audit, the facilitator should organize and coordinate the initiative in conjunction with the HD, starting from the planning, conduction and preparation of the final report, by involving colleagues about results and changes to be made in the process. This is because the audit is perhaps the branch with the highest clinical content. In the health technology assessment (HTA) field the facilitator could act as a liaison between the department, the HD and the DG, collaborating in the new technologies or new therapeutic/clinical pathways assessment, to identify the organizational impact; he cooperates with colleagues in the HTA projects development within the department, inter-department and with the HD. In the risk management field he works to spread awareness of the issues and to encourage change. The reporting of Quality Systems for the Evidence-Based Practice and Policy and the knowledge of the budget process should instead be mastered by everyone and at all levels, nevertheless the facilitator should strongly act to optimize these aspects: planning of meetings with budget experts, conveying projects, with the department and the HD, from a simple informative brochure for patients to initiatives for the coordination between in-hospital and out-hospital care. Therefore, this figure should act within the department to facilitate and simplify the horizontal interaction among other similar corporate structures and should help the Management to improve relations between hospital and territory, identifying the critical issues and possible solutions. In the discussion of the budget he should alert colleagues and directions, for this reason it is not reduced to a passive top-down process, but also has connotations of the bottom-up.

\section{Role of scientific societies}

The role and mission of scientific societies, such as the Federation of Associations of Hospital Doctors on Internal Medicine (FADOI), is to promote innovation and culture. The teaching and dissemination of the Clinical Governance is definitely in this direction and is in fact an issue that did FADOI own. To facilitate the acquisition of a culture of Clinical Governance in two-year periods 2008-2010 and 2010-2012, in collaboration with the University Carlo Cattaneo (LIUC), the University of Florence (the second edition) and the Institute of Health (ISS) has established a Master's Degree in Clinical Governance for the Internal Medicine. Seventy-one internists from all over Italy have thus had the opportunity to learn the tools of the Clinical Governance and thus contribute to spread this cultural approach in Internal Medicine of Italian hospitals. Also through its Permanent Area of Clinical Governance, FADOI promotes the assembly of its affiliates most affected on these subjects in order to help create the cultural climate suitable to face current and 
future economic problems and health where the internal medicine plays a fundamental role, and to grow, train and to relate the professionals who will assume the role of facilitators.

\section{The extension of the project to other departments}

In our proposal each department or each area should have a CG facilitator (for a maximum of 2-4 facilitators per hospital) that comes into staff as consultants to the HD, which in turn may use this figure as a mediator/facilitator in the diffusion, implementation and control of right application of CG tools. ${ }^{9}$ In this manner the internist would be the facilitator for the medical area, while other specialists with competence in CG would be assigned to other areas to create a small board of CG enriched with key figures (pharmacist, staff, management, etc.) as an engine of new ideas and for answering more quickly to the problems of inefficiency and low quality. However, the project should be extended to other departments cautiously, taking first of all into account the presence of heterogeneous specialties, for example otolaryngology with the oculistic (it is hard to imagine an otolaryngologist who coordinates an audit of cataract!); it is also important to avoid allocating this role to specialists not motivated or at the end of career, first of all, not to waste the skills of senior doctors more adequate to ensure high technical performances and secondly not to diminish the role of such a complex and fundamental figure as the facilitator.

\section{Potential application problems}

In addition to the potential obstacles already mentioned in the previous paragraphs we want to put the emphasis on some elements that can be a further impediment to the realization of the project. Among these we highlight issues like the distance, even geographical, and the fragmentation among specialties that may discourage cooperation, this in analogy to what is happening for the realization of Hospital organized for intensitivity of care. Prerequisite for overcoming these obstacles is a strong mandate from DG and HD that must support in a concrete way the project to ensure a real and not just institutional and regulatory commitment. It is mandatory to precisely define the role, tasks, areas of responsibility of the Facilitator, that should just assist the Department Directors, in order to avoid confusion in the roles and areas of overlap not only with the Director of the Department but also with the Medical Director, the Office of Education, etc. It is equally imperative that the facilitator of CG has specifically dedicated time to achieve the goals of which he is responsible and in order to avoid that this role will turn into another heavy and impossible task to accomplish.

\section{References}

1. Federspil G. Filosofia della Medicina. Milano: Raffaello Cortina Editore; 2008.

2. Morin E. I sette saperi necessari all'educazione del futuro. Milano: Raffaello Cortina Editore; 2001.

3. Scandellari C. La strategia della diagnosi. Padova: Piccin; 1981.

4. Nardi R, Scanelli G, Borioni D, et al. The assessment of complexity in internal medicine patients. The FADOI Medicomplex Study. Eur J Int Med 2007;18:283-7.

5. Ministero della Salute. Criteri di appropriatezza clinica, tecnologica e strutturale nell'assistenza del paziente complesso. Quaderni del Ministero della Salute 2013; 23:1-133.

6. Cicchetti A. L'organizzazione dell'ospedale. Fra tradizione e strategie per il futuro. Milano: Vita \& Pensiero Editore; 2002.

7. Hood L, Balling R, Auffray C. Revolutionizing medicine in the 21st century through systems approaches. Biotechnol J 2012;7:992-1001.

8. Scaly G, Donaldson L. Clinical governance and the drive for quality improvement in the NHS in England. BMJ 1998;317:61-5.

9. Nardi R, Arienti V, Nozzoli C, Mazzone A. Organizzazione dell'ospedale per intensità di cure: gli errori da evitare. Ital J Med 2012;6:1-13. 